\title{
Training Mode of Outstanding Doctors of Clinical Medicine by Distance Education
}

\author{
Shikai $\operatorname{Jin}^{1, \text { a * }}$ \\ ${ }^{1}$ Affiliated Hospital Provost's Office, Beihua University, Jilin, China \\ a254519852@qq.com
}

\begin{abstract}
Keywords: Training mode; outstanding doctors; clinical medicine; distance education
Abstract. With people demand medical services for higher and higher requirements, the demands of medical health structure reform and requirements of the high-level medical services. The training mode for outstanding doctors majoring was established through studying on current situation of clinical medical education, analyzing shortages of original mode by distance education. The training mode is described and it shown that special major with distinctive characteristics, good style of study, leading in our province, advanced in China.
\end{abstract}

\section{Introduction}

With the social development and technological progress, the medical model from the biomedical model into a bio - psycho - social medical model, marks the center of health and disease as medical science, has entered a new period of development, promote the transformation of medicine development in various fields, accelerate prevention, updated diagnosis and treatment technology, so that the whole of medical science face a fundamental change occurred. Medical continues to expand each branch and between disciplines intersect each other causing a constant derived from a large number of interdisciplinary and multidisciplinary medical curriculum subcommittee is getting smaller, new technologies are emerging, problems encountered when doctors are increasingly confronted with a patient diversification, to cultivate medical health personnel put forward higher requirements, medical education is facing new challenges.

In 1910, American educator Abraham Flexner published "Flexner Report", marking the beginning of subject-based medical curriculum, opened the first round of medical education reform wave, promoted the modern medical education changes, medical education completed university academic center, with modern medical science, based on the establishment of the first generation core curriculum reform; the 1960s, part of the medical colleges in the United States has carried out organ system-based multi-disciplinary comprehensive education reform, the introduction of the "problem-based learning" teaching, innovative curriculum formation of a new comprehensive system of international medical education curriculum reform had a significant impact, marking problem-oriented learning and integration of curriculum reform as the main content of the second generation of reforms; in 2010, setting off a global medical education third round of a new wave of reform of medical education, medicine global independent Board of Education in the "Lancet" published a paper entitled "New Century Health Experts: implement transformation of education, strengthen the interdependent world of health systems and services, "the report, presented on the basis of discipline-based university education, problem-oriented integration of two generations of reforms on education, promoting the transformation of learning, started with the system as the center, draw global experience, targeted to establish job competency requirements, improving the overall health system performance of the third generation of medical education reform new ideas.

2003 World Federation of Medical Education (WFME) officially announced the "Undergraduate Medical Education Global Standards", 2005 World Health Organization (WHO) and the World Federation of Medical Education (WFME) jointly issued the "Guideline for the Certification of undergraduate medical education," pulled from the global quality certification prelude medical colleges. To meet the trend of medical education to reposition and reform and development, the World Federation of Medical Education (WFME) to develop a "global standard undergraduate medical 
education", and soon after, the International Medical Education Society (IIME) also developed a "global basic medical education requirements ", the two gradually become the basis for quality assessment and Medical Education Reform and Development. China promulgated the "Chinese undergraduate medical education standards-clinical medicine specialty (Trial)" in 2008, the standard to international standards of medical education as a reference, combined with China's basic national conditions, taking into account the country's cultural traditions, educational system and educational resources and other factors to five years undergraduate clinical professional for applicable objects from the ideological and moral and professional qualities, knowledge of the target, skills, goals three clear objectives to be achieved graduates.

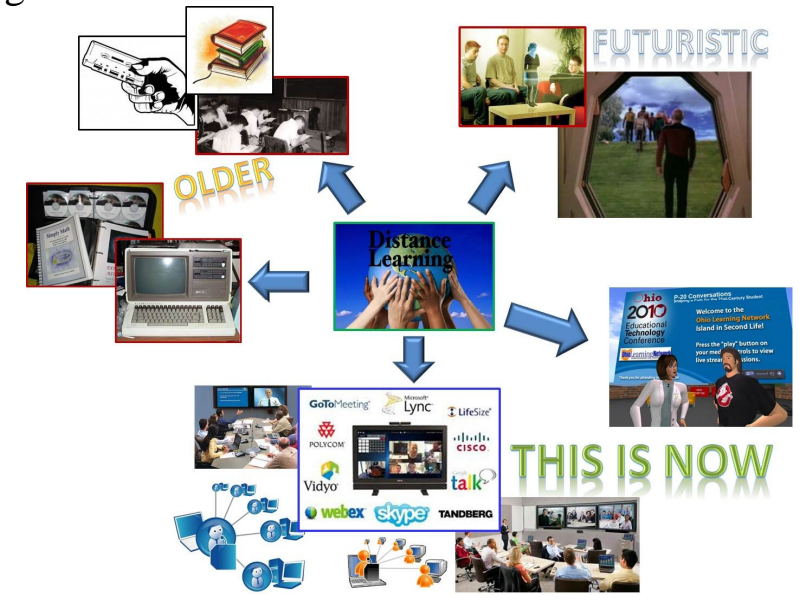

Fig. 1 International Network Classroom in Fig.

\section{Excellent physician training program}

In the domestic and international situation, driven by the Ministry of Education in recent years has issued a series of documents aimed at promoting higher medical education to go to improve the quality of teaching as the core content development. 2010 "Long-term Education Reform and Development Plan" release, explicitly proposed to start the implementation of the "excellent physician training program", in December 2011 the National Working Conference on the reform of medical education, further pointed out the direction of reform of medical education, raise to train medical personnel to be outstanding by deepening the reform of medical education, innovation and institutional mechanisms, and put forward the basic idea of the comprehensive reform of medical education. "Some Opinions of the Ministry of Education to improve the quality of higher education on a comprehensive" (high resolution [2012] 4 Sect) that "to promote comprehensive reform of medical education, the implementation of outstanding physician education and training program, exploration of clinical medical training model adapted to national health care reform needs . "May 2012, the Ministry of Education, the Ministry of Health jointly issued a document," Ministry of Education, the Ministry of Health on the implementation of outstanding physician education and training plan view "(teaching high [2012] No. 7), the official organization and implementation of" excellent physician education and training program " accelerating the comprehensive reform of clinical medical education, physician excellence culture a comprehensive plan and propose specific targets. Among them, the construction of the content of the first is "to carry five-year clinical training model reform." Around how to reform medical education model, train medical personnel excellent discussion also kicked off across the country.

\section{Five-year clinical medicine doctor training in basic guiding ideology of excellence}

(1) In order to deepen the reform as a driving force. Changing Ideas, enhance quality awareness, reform-minded; to organize professional certification, building teaching capacity development center focused on work as the starting point in training mode, curriculum content, methods, means and 
appraisal reform as the focus; to enhance inter-disciplinary integration, cross integration and collaborative innovation initiative, enthusiasm, and promote basic and clinical transformation and integration, promote the integration of clinical medicine and public health, promote the integration of the humanities and medicine, and to promote the integration of medical education and community health care services.

(2) The adaptation of health reform as the main line. Medical Education "a school across the two industries, two industries is the people's livelihood." In the health system and the education system to promote the depth of the environment, became a doctor to change the overall situation, firmly establish the concept of a health care reform medical education and services, to achieve "two around": around the progressive realization of "universal access to basic health care services" target advance medical talent training mode reform; health care reform around the deep-seated problems, and actively explore the education reform, the formation of medical education initiative to adapt to the development of medical and health situation.

(3) The competent job as a guide. Around medical graduates' job competency "demand-driven, medical and health sector needs to adapt to the medical personnel, efforts to optimize the type of talent structure, speed up the training of high-quality, application-oriented medical professionals.

(4) Ability to focus. Adhere to medical education, moral education first, as the most important capability. Vigorously strengthen the training of professional quality education and medical students caring spirit and interpersonal communication skills to medical students professional ethics, professional ethics and professional attitude as the basic content, allowing medical students have care of patients, respect for others, respect for life, teamwork good Deepening clinical practice teaching reform, promote the reform of teaching content and practice models, strengthen practice teaching, early clinical, more clinical, repeated clinical, improve medical students' clinical comprehensive clinical thinking ability and resolve practical problems; professionalism.

\section{Five-year clinical medicine physician training goal of excellence}

China Medical Education from the ideological and ethical standards and professional quality (12), knowledge of the target (10), the ability to target (13) three 35 defines the basic requirements for graduates to be achieved, raised need to be professional, the necessary Basics (natural sciences and clinical disciplines), clinical thinking and skills, communication skills, prevention of conception (crowd concept, health promotion and health education), the scientific method and critical thinking, lifelong learning and other capabilities of seven areas.

In accordance with the requirements of the standard of medical education, medical education for our shortcomings, combined with "excellent doctor" cultured "ethics" and "ability of clinical practice," the core elements, establish five-year clinical medicine doctor training in excellent overall objective is " Strong humanities, thick foundation, heavy skill, high-quality ", aims to cultivate good professional ethics, solid scientific knowledge of human nature, deep basic medical knowledge, a strong ability in clinical practice, clinical analysis and thinking skills, Medical graduates have the basic research and innovation ability, good self-learning and lifelong learning.

"Excellent Doctor" training to the competence-oriented, competency refers to the sum of those associated with the actual work situation, to promote individual and organizational development of knowledge, skills, attitude and all other personal traits. Around post competency, from the "knowledge, ability and quality" three excellent doctors to establish specific goals Talents: Knowledge objectives: undergraduate level clinical medical education system, learning the basic theory and basic knowledge of medicine, by the diagnosis of human diseases, basic training in treatment and prevention, master the basic medicine, clinical medicine, preventive medicine basic theory, basic knowledge, with human disease etiology, pathogenesis, classification ability to make identification. 


\section{Summary}

Study of "the competence-oriented" design ideas, focusing Dr. Yu Zhuoyue training mode reform, the masses gather intelligence on the health needs of the service, cohesion in student professionalism and clinical practice ability, highlight a core, two priorities i?? A core, that is to train students of professional ethics and the clinical practice ability as the core; two priorities, namely to reform the training system and excellent physician management system and mechanism innovation as the focus. From the philosophy of education, training objectives, curriculum, teaching content, teaching organization forms and methods, teaching staff and other aspects of performance appraisal system, to explore possible ways of supporting the comprehensive reform and innovation, initially built with "strong humanities, Training System theoretical models thick foundation, heavy skill, high-quality "for the training objectives.

Characteristics and practical conditions Medical Education of Medical Professional Training based, innovative management mechanism and mode of operation, set up a goal and two starting point, teaching quality guarantee system of the three platforms. That certification standards in medical education as the goal, the theory and practice of teaching the two starting point, the theoretical teaching (Department), experimental teaching (laboratory) and practical teaching (clinical departments) is "three platforms."

This study targeting accurate, effective for the current majority of medical humanities education is weak, lack of innovation, practical ability is not strong, self-learning ability and poor, by optimizing the curriculum, teaching practice optimization, optimization Professional Ethics Education Teaching Methods optimize the assessment and evaluation, better adapt to the development and reform of education and teaching reform of medical and health situation requires. Related research of this subject is still in its infancy, and improves the organization and management, selection of materials, guarantee the quality of teaching and many other problems still need to continue to explore and improve our reform practice.

\section{References}

[1] Ma Jing, IN TRADITIONAL FORCES of "excellent physicians' education and training. EDUCATION Wenhui, 2012, (2): 138-145.

[2] Liu Lijuan; Xu Jinsong; Wang Yue Global Minimum Essential Requirements in Medical Education and Evaluation Method. Northwest Medical Education, 2010,18 (1): 37-38.

[3] Zhu Hui-wide, Bobby Chao, Cao Dehe goods, and so on. Thinking about offering medical humanities and social science courses. China Higher Medical Education, 2001, (2): 60-71.

[4] Qin Nian Medical Curriculum System Reform of University of Hong Kong dynamic. Shantou University Medical College, 1998,11 (Suppl): 78-83.

[5] Sun Dan, Chen Jiqiang, Weyl clear, and so the status quo and prospect of Zhejiang University Course Construction of Basic Medical integrate network security.Ningxia Medical University, 2010,11 (z1):. 125-127.

[6] Zeng Jing preliminary exploration and feedback system integration curriculum reform in the system of medical education in eight years.Chinese Journal of Modern Medicine, 2010,20 (22): 3516-3518.

[7] Shu Tao efforts to develop an international perspective to the organ systems-based integrated medical curriculum.China Higher Medical Education, 2011, (7): 54-56.

[8] Li Yi Sha, left Xiaoxia, Luo Hui, et PBL teaching feedback and questions in medical education in the eight-year analysis. Chinese Journal of Medical Education, 2011, 10 (10): 1171-1173. 